\title{
Large Area Imaging Detector for Long-Range, Passive Detection of Fissile Material
}

\author{
Klaus P. Ziock, William W. Craig, Lorenzo Fabris, Richard C. Lanza, Shawn Gallagher,
} Berthold K. P. Horn, Norm W. Madden

\begin{abstract}
Recent events highlight the increased risk of a terrorist attack using either a nuclear or a radiological weapon. One of the key needs to counter such a threat is long-range detection of nuclear material. Theoretically, gamma-ray emissions from such material should allow passive detection to distances greater than $100 \mathrm{~m}$. However, detection at this range has long been thought impractical due to fluctuating levels of natural background radiation. These fluctuations are the major source of uncertainty in detection and mean that sensitivity cannot be increased simply by increasing detector size. Recent work has shown that this problem can be overcome through the use of imaging techniques. In this paper we describe the background problems, the advantages of imaging and the construction of a prototype, large-area $\left(0.57 \mathrm{~m}^{2}\right)$ gamma-ray imager to detect nuclear materials at distances of $\sim 100 \mathrm{~m}$.
\end{abstract}

\section{INTRODUCTION}

UCLEAR material cannot be detected at long range us1 ing traditional methods because its presence is masked by background radiation of unknown and spatially varying intensity [1]. Adding imaging to detection allows one to estimate the local background, making it possible to detect spatially localized radioactive sources from considerable distances.

To understand why imaging gives an order of magnitude or so improvement in performance in a passive search scenario, one must understand the limitations imposed by the use of an omni-directional, gamma-ray detector. We start by defining the rate observed in our detector at a random location in the region to be searched as the background, B counts $/ \mathrm{m}^{2} / \mathrm{keV} / \mathrm{sec}$. If we then use the same detector and move it around our search area, we find that $\mathrm{B}$ varies by as much as a factor of five from one place to another. Suppose a source of strength, $\mathrm{S}$ counts $/ \mathrm{keV} / \mathrm{sec}$ is to be found. To minimize the search time, we try to detect the source from as far away as possible. The source is "detected" when the signal from it exceeds the noise by some fixed ratio-e.g. when the signal is five times as strong as the noise.

If the background were a constant, we would write the signal-to-noise ratio (SNR) as:

Manuscript received October 29, 2003. This work was performed under the auspices of the U.S. Department of Energy by University of California, Lawrence Livermore National Laboratory under Contract W-7405-Eng-48.

K. Ziock, W. Craig, L. Fabris and N. Madden are with Lawrence Livermore National Laboratory, Livermore, CA 94550 USA (Corresponding author, K. Ziock, telephone: 925-423-4082, e-mail: ziock1@1lnl.gov).

R Lanza, B. Horn and S. Gallagher are with the Massachusetts Institute of Technology (telephone: 617-253-2399, e-mail: lanza@mit.edu, bkph@ai.mit.edu).

$$
S N R=\frac{s(r)}{\sqrt{s(r)+B}}=\frac{\frac{S A \varepsilon t}{4 \pi r^{2}}}{\sqrt{\frac{S A \varepsilon t}{4 \pi r^{2}}+A b(r) \varepsilon t}} \propto \sqrt{A t}
$$

where $A$ is the detector area, $t$ is the measurement dwell time, $s(r)$ is the signal, $b(r)$ is the local background rate and $\varepsilon$ is the detector efficiency. We find that longer dwell times and larger detectors give a better SNR and hence improve the search range. Unfortunately, this does not apply to the detection of weak emissions from nuclear materials in the field.

Equation (1) assumes that we are limited by counting statistics (i.e. we know the background, so the standard deviation of our measurement is the square root of the total number of counts detected.) But in practice, we do not know the spatially varying background and hence the appropriate expression is:

$$
S N R=\frac{s(r)}{B}=\frac{\frac{S A \varepsilon t}{4 \pi r^{2}}}{A b(r) \varepsilon t} \propto \frac{A t}{A t}=\text { Constant }
$$

In this case, the SNR is independent of both $A$ and $t$. As a result, neither a larger detector, nor a longer dwell time can improve the SNR. This is shown graphically in Fig. 1 where the (simulated) counts reaching a detector that tracks past a source at different distances of closest approach are shown for various detector sizes. (The closest approach occurs at $x=0$.) The broad increase in counts centered at $\sim x=25 \mathrm{~m}$ represents a factor of two increase in the background. As can be seen, once the detector is $20 \mathrm{~m}$ from the source, it cannot be distinguished from the broad background bump.

Imaging adds the ability to estimate the local background. One makes use of this information by looking for isolated sources that produce a slightly enhanced flux in a limited area of the image (i.e. only a few pixels are affected by the photon flux from the source.) The local, slowly-varying, background can be determined from the neighboring pixels in the image. This means we know the background and can use the SNR expression given by (1) instead of the one given by (2). For a very large detector, of the sort we are building, the background rate can be as high as $1000 \mathrm{cnts} / \mathrm{s}$ so that the improvement in SNR is $\sim 30$. In Fig. 2 we present results of the same simulation as in Fig. 1, but with an imaging detector. The figure clearly shows successful detection of the source at $50 \mathrm{~m}$ using a large detector. Based on these results we are building the large imager described below. 

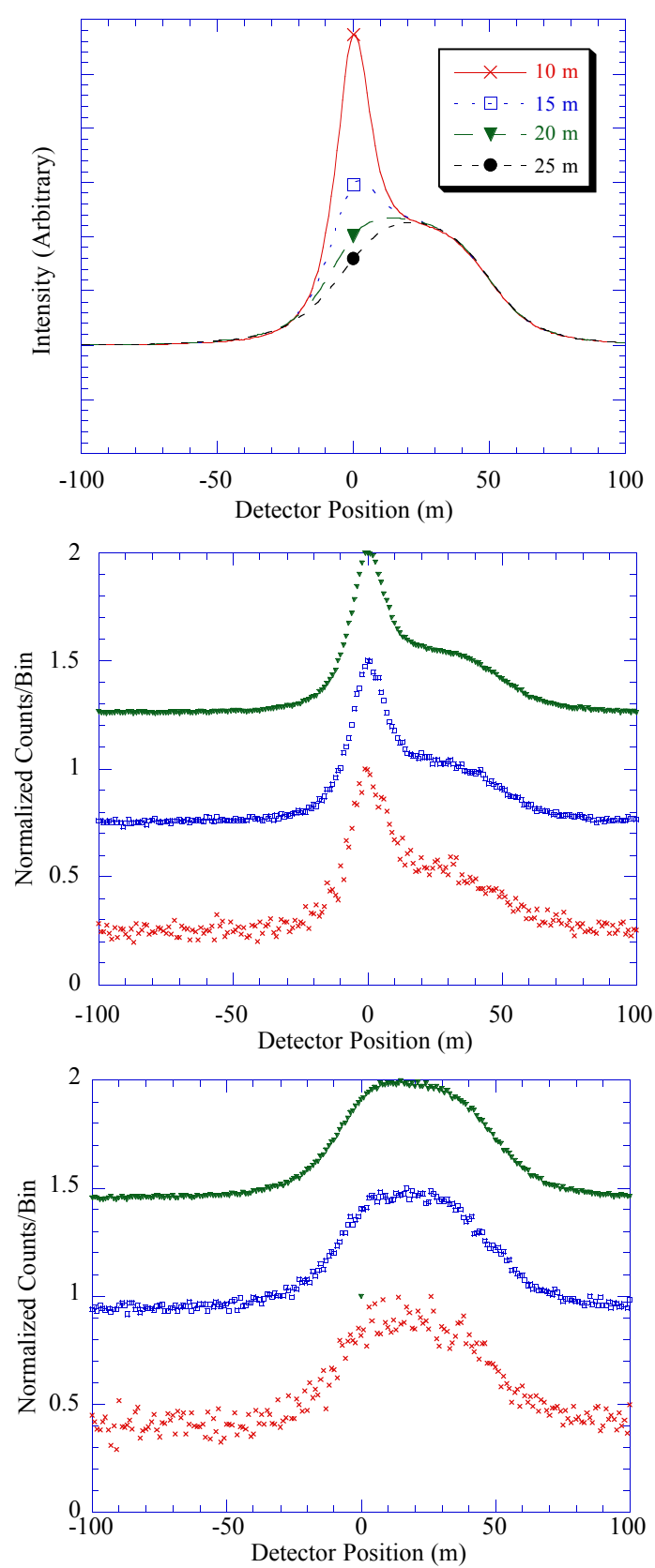

Fig. 1. Response of a detector passing by a fixed point source. The top plot shows the noiseless response for different distances of closest approach. The middle plot shows the response for detectors of 100,1,000 and 10,000 $\mathrm{cm}^{2}$ area (bottom to top trace, offset by 0.5 for clarity) passing the source at a distance of closest approach of $10 \mathrm{~m}$ and includes counting statistics noise. The bottom plot shows the response for detectors of 100, 1,000 and 10,000 $\mathrm{cm}^{2}$ area (bottom to top trace, offset by 0.5 for clarity) passing the source at a distance of closest approach of $20 \mathrm{~m}$ and includes counting statistics noise. While a larger detector improves the counting statistics, it clearly does not improve the range at which a source can be detected.

\section{LARGE IMAGER DESIGN}

Our large area imager is based on a one-dimensional, coded aperture design that uses existing $10 \times 10 \times 10 \mathrm{~cm}^{3} \mathrm{NaI}$ detectors. A photograph of the imager with one row of the detectors installed is shown in Fig. 3. The coded aperture mask is based on a 19-element, one-dimensional, URA pattern [2]. It is con-

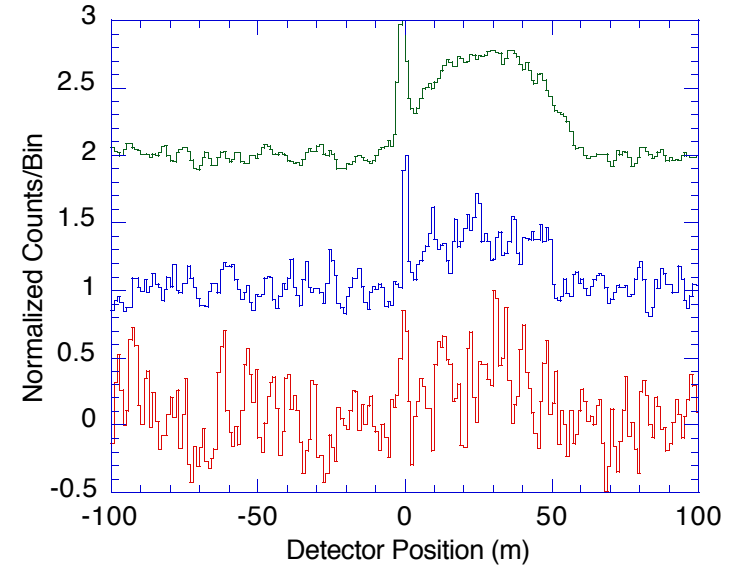

Fig 2. Normalized response of an imaging detector passing by a fixed point source. Bottom to top trace are for $100 \mathrm{~cm}^{2}$ detector at $30 \mathrm{~m}$ closest approach, 1,000 $\mathrm{cm}^{2}$ detector at $30 \mathrm{~m}$ closest approach and a $10,000 \mathrm{~cm}^{2}$ detector at $\mathbf{5 0}$ m closest approach. (The traces are offset by one for clarity.) Larger imaging detectors clearly help.

structed of Linotype metal (an alloy with $85 \% \mathrm{~Pb}, 11 \% \mathrm{Sb}$, $3 \% \mathrm{Sn}$, and $1 \%$ other materials) $4-\mathrm{cm}$ thick with a $10.8-\mathrm{cm}$ pitch imposed by the extent detectors. One-dimensional imaging was selected for ease of implementation and the proposed suburban, light-urban search application for the instrument. Long-range search in dense urban areas is extremely difficult due to the shielding effects of concrete floors in high-rise buildings of more than a few floors. Further motivation and design details are presented below.

\section{A. Monte Carlo Simulations}

A Monte Carlo code was developed to optimize the imager response and develop data handling algorithms. The code was designed to simulate the counts viewed by the system as it tracks past a source. It includes such effects as atmospheric attenuation, finite mask thickness, number of mask elements, detector velocity and variable collimation. The location of the source along the track, the distance of closest approach and the source strength are all variable quantities so that different problems can be run. In addition, the strength of the background is a free parameter that includes a constant and/or modulated (sinusoidally with position) fluence.

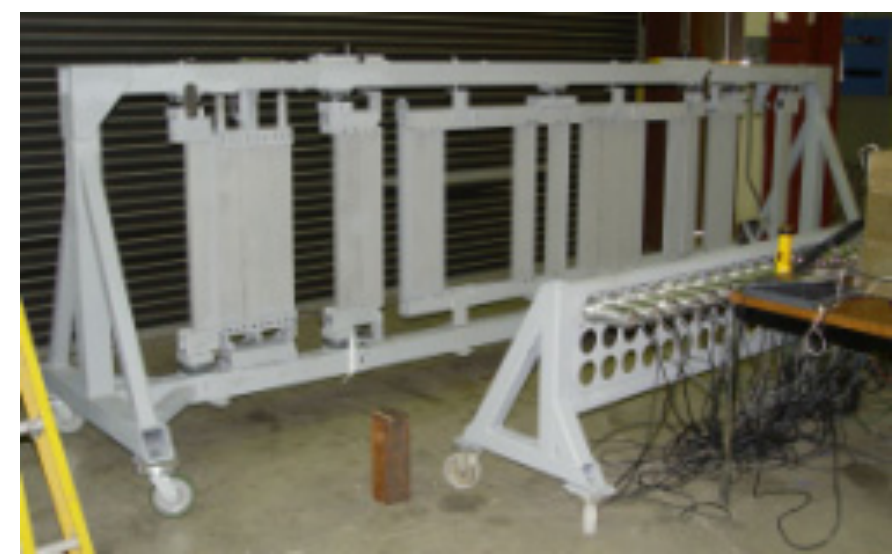

Fig. 3. Picture of the imager showing the mask and the detector array. Only the top row of the $19 \times 3$ detector array is installed. 


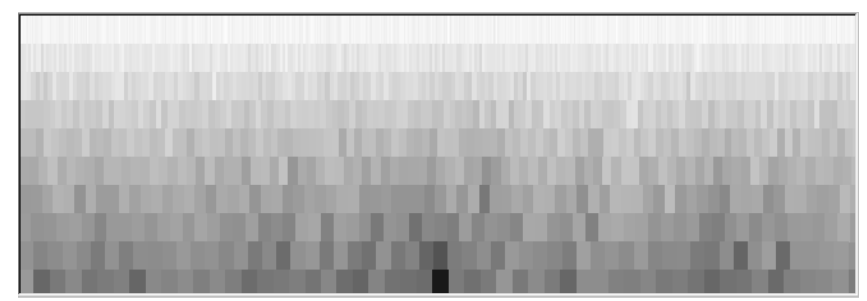

Fig. 4. Sample scan past a $1 \mathrm{mCi}{ }^{137} \mathrm{Cs}$ source at $95 \mathrm{~m}$ distance of closest approach, at a speed of $40 \mathrm{~km} / \mathrm{hr}$ for a $0.57 \mathrm{~m}^{2}$ detector area and a background rate of 500 counts $/ \mathrm{sec} / \mathrm{m}^{2}$. The distance from the imager increases from the top to the bottom of the image in $10 \mathrm{~m}$ steps. The source is clearly visible at the bottom of the image.

\section{1) Simulation Implementation}

In the code, the position of the detector imager is stepped in one millisecond increments. During each step the flux from a point source at a definable distance and location of closest approach is calculated. The calculation of the expected count is based on the source strength, the detector solid angle at the current location and atmospheric attenuation. A Poisson generator [3] is used to select the number of counts incident on the detector during the step. These are randomly distributed across the detector face. Based on the event location and the geometry to the source, the mask elements that the photon traverses at the front and back of the mask are calculated. If either of these is opaque, then the event is rejected. (This simulates the effects of mask thickness.) In addition, slat collimators of selectable length between the individual pixels are used to impose a collimation function. If an event survives all of these obstacles, then it is recorded in the correct bin of one of 10 simulated detector arrays that are used to track counts for different distances of closest approach (which is not known in advance.) These 10 detector arrays are defined for bins of closest approach that are $10 \mathrm{~m}$ deep, centered at $5,15,25, \ldots, 95 \mathrm{~m}$. As the detector array position is incremented, a check is made to see if the detector location equals or exceeds the edge of one of the corresponding ten fixed world-pixel maps. These maps are correlated with the world-pixel size for each distance bin determined from the angular resolution of the imager (mask pixel size divided by the focal length times the average distance of

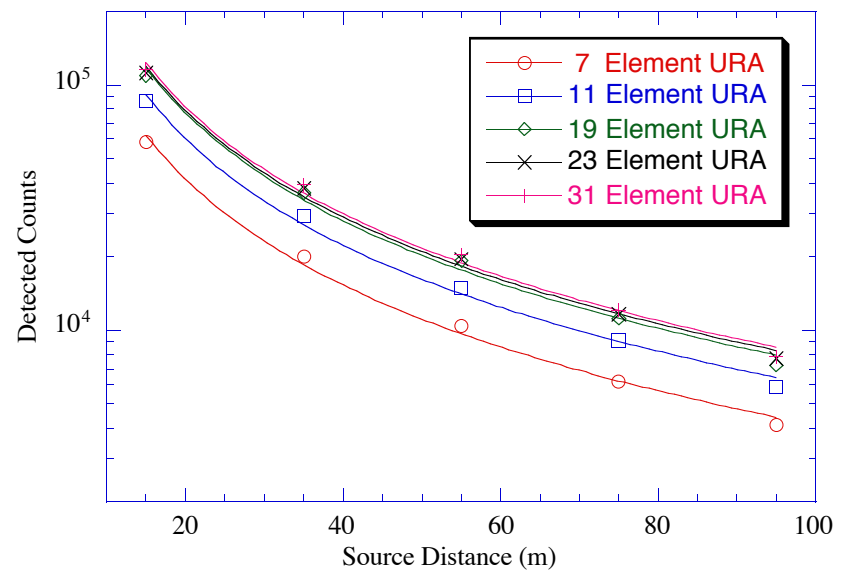

Fig. 5. Detected counts versus source distance of closest approach for different fields of view as represented by the number of elements in the coded aperture pattern. closest approach). If so, then the data in the detector array for that distance is used to generate an image and the image is added to the appropriate world pixel map. The detector at that distance is zeroed and the system continues.

\section{2) Simulation Results}

Sample results from the simulations are shown in Fig. 4 where the images of a scan at $40 \mathrm{~km} / \mathrm{h}$ past a one millicurie ${ }^{137} \mathrm{Cs}$ source are shown. Relatively high backgrounds of 500 $\mathrm{cnts} / \mathrm{m}^{2} / \mathrm{sec}$ were used in the simulations. The source is clearly visible in the bottom center of the image. The distance of closest approach was $95 \mathrm{~m}$. The distance to the source is primarily determined from the change in parallax as the data in the different distance-of-closest-approach detector arrays are mapped to the corresponding world pixel maps.

The code was used to optimize the imager with restrictions placed on the parameters by the fixed size of the existing detector elements, a maximum focal length of one meter and a desire to minimize the overall system length.

The first simulations were used to select the best imager length, which is determined by the number of elements in the mask pattern. The results are shown in Fig. 5 where we plot the detected (maximum) signal versus the distance of closest approach to the source and the number of elements in the coded aperture pattern. As one adds mask elements of fixed width, the field of view of the imager increases so that a source is in the field of view for a longer period. (Full collimation to one cycle of the mask pattern was used in the simulations.) As can be seen, the advantage gained from more pixels diminishes as the field of view is increased beyond $\sim 45$ degrees. At large angles, the projected detector area and the long range to the source mean that few events are obtained and only small increases in sensitivity will occur beyond 19 pixels. The results were found to hold down to the lowest lines of interest at $100 \mathrm{keV}$, indicating that atmospheric absorption did not significantly limit the performance at wide fields of view.

A second set of simulations was used to determine whether collimation was needed. For a fully encoded imager with the mask twice the size of the detector, images of objects that walk off of one side of the field of view will wrap or suddenly appear on the other side of the decoded image as the source continues to move out of the field of view. This is a result of partial mask patterns projected by the source and is of concern in our test imager. One solution to this problem is to restrict the field of view of a single detector pixel to one cycle of the mask pattern (19 pixels) using slat collimators between the

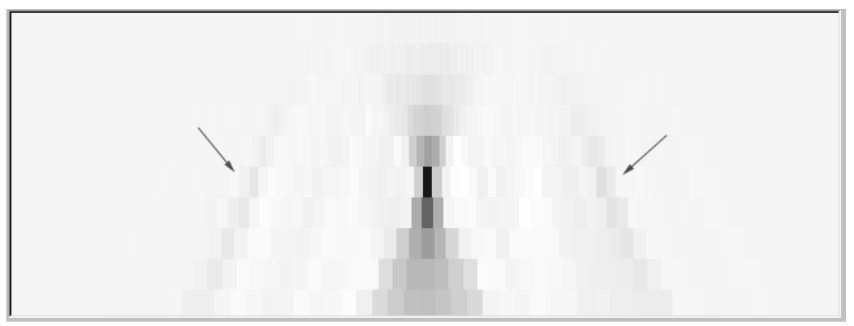

Fig. 6. Scan with no background showing the ghost peaks before and after the main source (at arrows). The data represents a $1 \mathrm{mCi}$ source at $55 \mathrm{~m}$ closest approach for a $1 \mathrm{~m}^{2}$ detector traveling at $40 \mathrm{~km} / \mathrm{hr}$. 


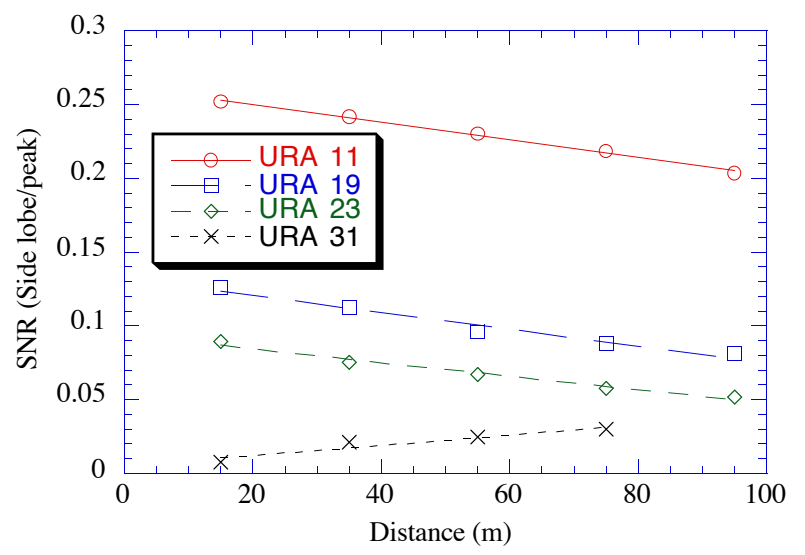

Fig. 7. Size of the ghost peak due to the lack of collimation as a fraction of the main peak height for different numbers of mask elements.

pixels. However, this reduces the flux from the source as it transits the field of view and also adds weight and complexity to the imager. Simulations with no collimation beyond that inherent in the 4-cm mask thickness were run. The results (Fig. 6) show that a strong point source will have a weak ghost peak before and after the main peak. The size of the peak varies with the number of mask elements as shown in Fig. 7. The small side peaks of our 19-element coded aperture were not considered worth suppressing because of the loss in sensitivity that would result from additional collimation.

In imaging objects at a finite distance, one must include the geometric projection effects that magnify the mask pattern at the detector [4]. A source at a distance of closest approach, $b$, will magnify the mask pixel, $a$, by a factor

$$
\frac{a^{\prime}}{a}=\frac{b+f}{b}
$$

where $f$ is the distance between the mask and the detector. Magnification factors differing from one by a few percent can cause artifacts in the decoded image. Although these are mostly washed out as a source transits through the field of view in a scan, there is a net loss in efficiency since contrast in the shadow pattern is lost. Ideally, one would use detectors without mechanically defined (fixed) pixel sizes (as was done in the simulation with the 10 different detector arrays each representing different binning of the same data.) As this was not possible here, we chose to optimize the system for distant sources and matched the mask pattern pitch to the detector pitch. This sacrifices some of the sensitivity to nearby sources, however, these sources are already the most detectable because of the higher count rate.

An additional complication posed by the use of the existing detectors was the large individual pixel size. Ideally, to avoid aliasing effects, one oversamples the mask pattern, using a detector pixel pitch half that of the mask pixel pitch [5]. This would mean mask pixels of the order of $20 \mathrm{~cm}$, leading to an unwieldy focal length for the desired $\sim 5 \mathrm{~m}$ pixel size at the maximum range. In addition, a coded aperture pattern of 19 pixels means a 38-pixel mask that would be almost 8 meters long, requiring an unrealistically long vehicle. The 4 meter
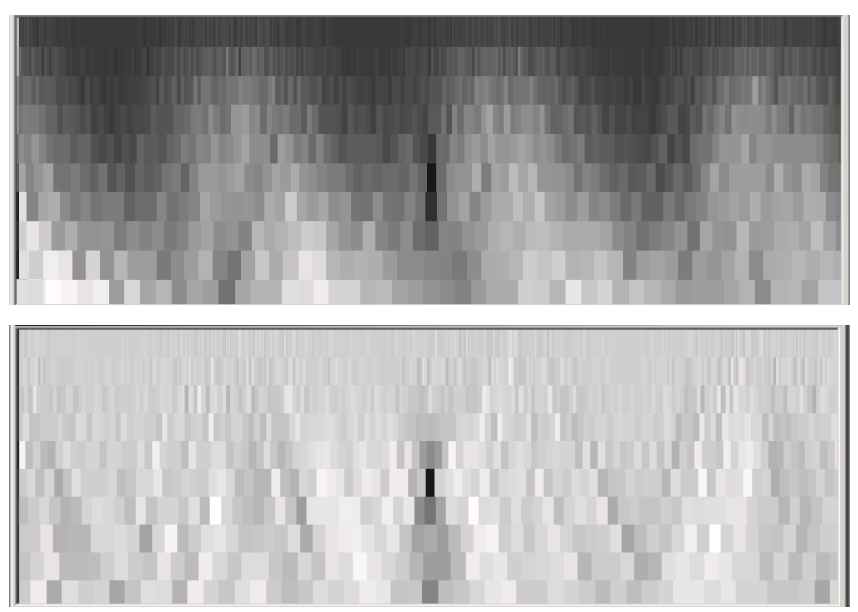

Fig. 8. Source at $55 \mathrm{~m}$ scanned in two passes, to simulate the mask/antimask process. The upper panel shows only the mask pass, the bottom shows the combined data. A background of 500 counts $/ \mathrm{sec} / \mathrm{m}^{2}$ with a sinusoidally modulated background of another 500 counts $/ \mathrm{sec} / \mathrm{m}^{2}$ and a wavelength of $25 \mathrm{~m}$ is used. The source is $0.5 \mathrm{mCi}$, the detector size is $0.5 \mathrm{~m}^{2}$ and the scan velocity is $56 \mathrm{~km} / \mathrm{hr}$. The combined data is equivalent to a single pass with twice the background and twice the detector area.

length of the mask with the one-to-one sampling we use is another reason to desire smaller pixel sizes.

A technique that significantly enhances the robustness of coded aperture imaging is the use of sequential, equal-time, mask and anti-mask acquisitions. By interchanging open and closed elements of the mask pattern, one can remove spurious variations in counts versus position in the detector [4]. Such variations are inevitably present and lead to artifacts in the image. In the scanning system described here, use of this technique is problematic. A second option, to simultaneously obtain the data with two vertically stacked systems was considered. Due to the different radiation environments of imagers placed one above the other, a serial approach was deemed better. Unfortunately, following a mask imager by an anti-mask imager would result in an unacceptably long instrument $(8 \mathrm{~m})$ due to the fixed detector pixel size. As a proof-of-principle instrument for the imaging technique, we opted to take separate scans past the same source field, once with the mask pattern and a second time with its inverse. To effect the change, a number of mask elements are rotated about axes of antisymmetry, while others are raised or lowered into or out of the detector field of view.

The great advantage of the mask/anti-mask technique is shown graphically in Fig. 8 where we impose a leakage background (unmodulated by the mask) that varies sinusiodally with detector position. The varying background that dominates the system response in the mask-only data, shows only as increased statistical noise in the mask/anti-mask decoded image.

\section{B. Mechanical Design}

Based on the results of the simulations, an instrument using a one-dimensional 19-element-base, coded aperture pattern was constructed. The mask elements are made of Linotype metal. This provides almost the density of lead but is structurally superior. The mask elements are $75 \mathrm{~cm}$ high to provide a verti- 
cal field of view designed to cover a two-story building at 6 $\mathrm{m}$. Their 4-cm thickness provides high contrast for gamma-ray energies up to $3 \mathrm{MeV}$. The elements are captured in a steel frame as shown in the Fig. 3. The system can be rapidly reconfigured from mask to the anti-mask configuration.

The detectors are mounted to a plate that is held in a similar, although smaller, frame. Each of the 19 pixels is comprised of three of the $10 \times 10 \times 10 \mathrm{~cm}^{3}$ detectors stacked vertically. To protect the $\mathrm{NaI}(\mathrm{Tl})$ detectors from thermal shock the instrument is mounted in the rear of a refrigerated truck.

\section{Electronics}

A custom 64-channel data acquisition system (one channel for each scintillator-photomultiplier (PMT) detector) is under construction to handle data from the system. The PMTs are operated at a nominal gain of $10^{5}$. The event generated charge from a PMT is converted into a voltage by a local capacitor connected directly to the anode at the PMT base. A voltage amplifier also connected to the PMT anode, sends the signal to the processing electronics.

The system is built around a gated integrator with a parallel fast channel. A discriminator in the fast channel recognizes an event and initiates the signal processing cycle in the gated integrator. Voltage signals from a PMT are converted back into a short charge pulse and integrated for $2.2 \mu \mathrm{s}$. The integrated signal is then converted by a low-power, 16-bit ADC and decimated to an 11-bit word. This provides near nuclear quality conversion without the use of dithering.

Every ADC generates an interrupt signal when it has valid data. The interrupt signals are decoded by a priority encoder and serviced by the steering logic. The ADC data is combined with the PMT number to from an event word. This is stored in a FIFO buffer. To allow a world-image map to be generated, the logic also generates periodic time stamp events and imager location events (the latter, through decoding of a fifth wheel attached to the vehicle.) These are also stored in the FIFO buffer. A fast PCI interface (NI-PCI-6534) [6] is used to perform high-speed data transfer between the electronics and a personal computer.

The system is designed to withstand a sustained rate of $\sim 100 \mathrm{kcps}$ per channel. Total power consumption for the data acquisition system is $\sim 120 \mathrm{~W}$. It is designed to run off of the vehicle power supply. Several tests have been performed and the system shows resolution performance dominated by the detector statistics.

\section{PReliminary Performance}

The full electronics system is scheduled for completion late in 2003. To verify the imaging performance of the system, we have performed stationary tests using one row of detectors and a very early version of the acquisition system (originally constructed for a $19 \times 19$ Ge strip detector) [7].

In preparation for the tests, the gains of the detectors were approximately equalized by varying the gain of the preamplifiers and/or the high voltage, as required. Residual gain varia-

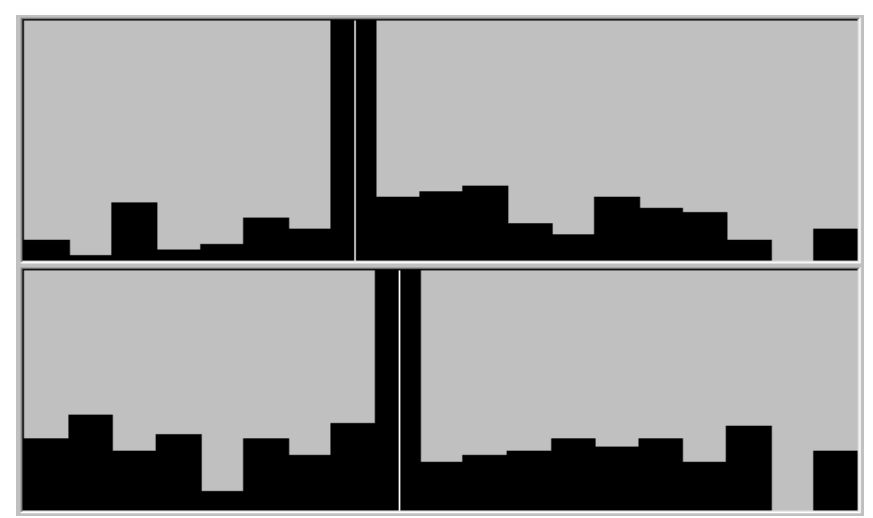

Fig. 10. Two images of $30 \mu \mathrm{Ci}$ source at 10 meters taken in one second with one third of the final detector area. The source is moved by one pixel between the two images.

tions were removed by an automated fitting routine that performed a chi-square minimization to Gaussian line profiles of data collected using a ${ }^{137} \mathrm{Cs}$ and a ${ }^{60} \mathrm{Co}$ source.

The calibrated imager was used with a shortened focal length of $36 \mathrm{~cm}$. This allows the use of weaker sources at closer range, while maintaining the pixel sizes. A first image of a point source is shown in Fig. 10.

\section{CONCLUSIONS}

Preliminary results from the instrument indicate that it performs as expected. The sensitivity to stationary point sources matches simulation results. Further tests are planned with the detector driven past fixed sources once the full electronics system is completed. In addition, the system will be used to measure the spatially varying and directional nature of the natural and man-made radiation environment.

\section{ACKNOWLEDGMENT}

The authors gratefully acknowledge the mechanical engineering support provided by T. Decker, the mechanical design and development work of D. Carter, and D. Carr and the contributions of summer students J. McDonald and E. Alexander. All are with LLNL. The authors also wish to acknowledge JeanFrancois Beche at Lawrence Berkeley National Laboratory.

\section{REFERENCES}

[1] K. Ziock, W. Goldstein, "The lost source, varying backgrounds and why bigger may not be better," in Unattended Radiation Sensor Systems for Remote Applications, J. Trombka, D. Spears, P. Solomon, editors, AIP Conf. Proc. vol. 632, pp. 60-70, 2002.

[2] S. Gottesman, E. Fenimore, "New family of binary arrays for coded aperture imaging," App. Opt., vol. 28, No. 20, pp. 4344-4352, Oct. 1989.

[3] W. Press, S. Teukolsky, W. Vetterling, B Flannery, Numerical Recipes in C, Cambridge Univ. Press, New York, NY, 1995. p. 294.

[4] K. Ziock, C. Hailey, T. Gosnell, J. Lupton, F. Harrison, “A gamma-ray imager for arms control," IEEE Trans. Nucl. Sci., vol. 39, no. 4, pp. 1046-1050, 1992.

[5] E. Caroli, J. Stephen, G. di Cocco, L. Natalucci, A. Spizzichino, "Coded aperture imaging in X- and gamma-ray astronomy," Space Sci. Rev., vol. 45, no. 3-4, pp. 349-403, Jan., 1987.

[6] National Instruments, http://www.ni.com/

[7] K. Ziock, N. Madden, E. Hull, W. Craig, T. Lavietes, C. Cork, "A germanium-based coded aperture imager," IEEE Trans. Nucl. Sci., vol. 49, no. 4, pp. 1737-1742, Aug., 2002. 